\title{
The flavonoid glabridin attenuates 2-deoxy-D-ribose-induced oxidative damage and cellular dysfunction in MC3T3-E1 osteoblastic cells
}

\author{
HYUN-SOOK KIM ${ }^{1}$, KWANG SIK SUH ${ }^{2}$, ARA KO ${ }^{3}$, DONGGEUN SUL ${ }^{4}$, DALWOONG CHOI $^{5}$, \\ SEUNG KWAN LEE ${ }^{1 *}$ and WOON-WON JUNG ${ }^{6 *}$ \\ ${ }^{1}$ Department of Biomedical Science, College of Health Science, Korea University, Seongbuk-gu, \\ Seoul 136-703; ${ }^{2}$ Research Institute of Endocrinology, Kyung Hee University Hospital, Dondaemun-gu, \\ Seoul 130-702; ${ }^{3}$ Department of Biomedical Sciences, ${ }^{4}$ Environmental Toxico-Genomic and Proteomic Center, \\ College of Medicine, Seongbuk-gu, Seoul 136-705; ${ }^{5}$ Department of Environmental Health, \\ ${ }^{6}$ Research Institute of Health Science, College of Health Science, Korea University, \\ Seongbuk-gu, Seoul 136-703, Seoul, Republic of Korea
}

Received August 6, 2012; Accepted October 11, 2012

DOI: $10.3892 /$ ijmm.2012.1172

\begin{abstract}
Reducing sugar 2-deoxy-D-ribose (dRib) produces reactive oxygen species (ROS) through autoxidation and protein glycosylation and causes dysfunction of osteoblasts. In the present study, glabridin, a natural flavonoid, was investigated to determine whether it could influence dRibinduced oxidative damage and cellular dysfunction in the MC3T3-E1 mouse osteoblastic cell line. Osteoblastic cells were treated with dRib in the presence or absence of glabridin. Cell viability, apoptosis, ROS production and mitochondrial membrane potential $\left(\Delta \Psi_{\mathrm{m}}\right)$ were subsequently examined. It was observed that $\mathrm{dRib}$ reduced cell survival and $\Delta \Psi_{\mathrm{m}}$, while it markedly increased intracellular levels of ROS and apoptosis. However, pretreatment of cells with glabridin attenuated all the dRib-induced effects. The antioxidant $\mathrm{N}$-acetyl-L-cysteine (NAC) also prevented dRib-induced oxidative cell damage. In addition, treatment with glabridin resulted in a significant elevation of alkaline phosphatase (ALP) activity, collagen contents and osteoblast differentiation genes [ALP, collagen, osteopontin (OPN), osteoprotegerin (OPG) and osteocalcin
\end{abstract}

Correspondence to: Dr Woon-Won Jung, Research Institute of Health Science, College of Health Science, Korea University, San 1, Jeongneungdong, Seongbuk-gu, Seoul 136-703, Republic of Korea

E-mail: woonun@korea.ac.kr

Dr Seung Kwan Lee, Department of Biomedical Science, College of Health Science, Korea University, San 1, Jeongneung-dong, Seongbuk-gu, Seoul 136-703, Republic of Korea

E-mail: seunggwan@korea.ac.kr

*Contributed equally

Key words: glabridin, flavonoid, osteoblastic cells, 2-deoxy-D-ribose, oxidative stress
(OC)] and bone morphogenetic protein (BMP) genes (BMP2, BMP4 and BMP7). In mechanistic studies of the antioxidative potential of glabridin, we found that glabridin activated $\mathrm{dRib}$-induced decreased expression of phosphatidylinositol 3'-kinase (PI3K) and protein kinase B 2 (AKT2) genes, which are master regulators of survival-related signaling pathways. Glabridin also upregulated the gene expression of antioxidant enzymes, superoxide dismutase 1 (SOD1) and glutathione peroxidase 4 (GPX4), which were inhibited by dRib. Taken together, these results suggest that glabridin attenuates dRibinduced cell damage in osteoblastic cells and may be useful for the treatment of diabetes-related bone disease.

\section{Introduction}

Oxidative stress results from a persistent imbalance between antioxidant defense mechanisms and the production of highly reactive oxygen species (ROS) (1). The chronic hyperglycemia leads to oxidative stress, which is involved in the progression of pancreatic $\beta$-cell deterioration as well as in the development of diabetic complications (2). Bone complications in diabetes include an early onset of osteopenia and osteoporosis $(3,4)$. These conditions lead to an increase in bone fracture and a delay in healing of fracture that affects the quality of life (5). In vitro studies have shown that oxidative stress inhibits osteoblastic differentiation (6) and induces osteoblast insults and apoptosis (7). One of the mechanisms of diabetes-related bone disease may be the direct effects on osteoblasts and bone turnover. An imbalance between bone-forming osteoblasts and bone-resorbing osteoclasts leads to the pathogenesis and etiology of certain bone metabolic diseases, including osteoporosis and osteopetrosis (8).

Sugars that contain aldehyde groups that are oxidized to carboxylic acids are classified as reducing sugars, and they produce ROS through autoxidation and protein glycosylation (9-11). The 2-deoxy-D-ribose (dRib) is a strong reducing sugar that becomes highly reactive with proteins $(12,13)$. 
Since glucose is the least reactive of the reducing sugars and requires long-term exposure time to provoke oxidative stress by the cells (11), we selected dRib as a surrogate for glucose to induce oxidative damage of MC3T3-E1 osteoblastic cells. We previously demonstrated that dRib promotes apoptosis by increasing oxidative stress in HIT-T15 pancreatic $\beta$-cells (12-15) and MC3T3-E1 osteoblastic cells (16-18).

Glabridin is an isoflavan compound, one of the major active flavonoids in licorice (19). It has been reported to exhibit multiple pharmacological activities such as cytotoxic activity (20), antimicrobial activity (21) and protection from oxidative stresses (22). Treatment of osteoblastic cells with glabridin prevented apoptosis and production of PGE2 and NO induced by TNF- $\alpha$ (23). Glabridin has been reported to protect osteoblastic cells against antimycin A-induced cytotoxicity (24), suggesting that the enhancement of osteoblast function by glabridin may result in the prevention of osteoporosis and inflammatory bone diseases. We recently reported on glabridin-induced prevention of osteoclastogenesis by inhibition of RANKL-induced activation of signaling molecules and subsequent transcription factors in osteoclast precursor cells (25).

Although there is some evidence that the health beneficial and pharmacological effects of glabridin are due to the activation of bone formation and the decrease in oxidative stress, no study has investigated whether glabridin can protect osteoblastic cells against oxidative damage mediated by dRib, as a source of ROS. Oxidative stress is involved in the modulation of the expression of transcription factors and cellular signaling, which may affect osteoblast functions. In the present study, we aimed to investigate the effects of glabridin on the oxidative stress-induced damage and cellular dysfunction in MC3T3-E1 osteoblastic cells.

\section{Materials and methods}

Cell culture. Murine osteoblastic MC3T3-E1 cells were obtained from the American Type Culture Collection (Rockville, MD, USA). Cells were cultured in $\alpha$-modified minimal essential medium ( $\alpha$-MEM; Invitrogen, Carlsbad, CA, USA) supplemented with $10 \%$ fetal bovine serum (FBS; Sigma Chemical Co., St. Louis, MO, USA), $100 \mu \mathrm{U} / \mathrm{ml}$ penicillin, and $100 \mu \mathrm{g} / \mathrm{ml}$ streptomycin. The cultures were maintained at $37^{\circ} \mathrm{C}$ in a humidified $5 \% \mathrm{CO}_{2}$ atmosphere and sub-cultured by trypsinization with $0.05 \%$ trypsin- $0.02 \%$ EDTA in $\mathrm{Ca}^{2+}$ - and $\mathrm{Mg}^{2+}$-free Dulbecco's phosphate-buffered saline (DPBS) when the cells reached approximately $70 \%$ confluence. For assessment of cell viability, apoptosis and ROS production, the cells were plated in 24-well culture plates at a density of $2 \times 10^{4}$ cells/well. Two days after culture, cells were treated with glabridin (0.01-50 $\mu \mathrm{M})$ for $24 \mathrm{~h}$ in $\alpha$-MEM containing $0.5 \% \mathrm{FBS}$. The cells were also seeded in 6-well culture plates at a density of $1 \times 10^{5}$ cells/well and treated with culture medium containing $10 \mathrm{mM} \beta$-glycerophosphate and $50 \mu \mathrm{g} / \mathrm{ml}$ ascorbic acid to initiate in vitro mineralization (26). Cell culture medium was changed every 2 days. After 6 days, cells were cultured with medium containing $\mathrm{dRib}$ and/or glabridin for 2 days to measure the alkaline phosphatase (ALP) activity, collagen content, and gene expression.
Assessment of cell viability. Cell viability was determined by measuring cell metabolic activity using the CCK-8 kit (Dojindo Co., Kumamoto, Japan) (27). The CCK-8 contains WST-8 [2-(2-methoxy-4-nitrophenyl)-3-(4-nitrophenyl)-5-(2, 4-disulfophenyl)-2H-tetrazolium, monosodium salt], which produces a water-soluble formazan dye upon reduction in the presence of an electron carrier. The amount of yellow formazan dye generated by the activity of dehydrogenases in cells is directly proportional to the number of living cells. Osteoblastic MC3T3-E1 cells were plated in 24-well cell culture plates at a density of $2 \times 10^{4}$ cells/well. At the end of the culture period, $50 \mu \mathrm{l}$ of the CCK- 8 solution was added to each well of the culture plate, which contained $500 \mu \mathrm{l}$ of the medium. Following a 4-h incubation, absorbance was measured with a Zenyth 3100 multimode detector (Anthos Labtec Instruments $\mathrm{GmbH}$, Wals/Salzburg, Austria) at $450 \mathrm{~nm}$ using a $650 \mathrm{~nm}$ filter as a reference. Cells incubated with culture medium alone were used to determine $100 \%$ viability and were included as a control in all the experiments to allow estimation of the percent viability of the cell samples.

Apoptosis determination by ELISA. A cell death ELISA kit (Roche Molecular Biochemicals, Mannheim, Germany), which quantitatively detects cytosolic histone-associated DNA fragments, was used to measure apoptosis according to the manufacturer's instructions. Briefly, cells were seeded at a density of $2 \times 10^{4}$ cells in 24 -well culture plates. The culture conditions used were the same as those described for the cell proliferation assay. Following incubation, cells were lysed and intact nuclei were pelleted by centrifugation. An aliquot of supernatant was used as the source of antigen for sandwich ELISA using a primary anti-histone monoclonal antibody that was bound to the streptavidin-coated wells of a microtiter plate. Subsequently, plates were treated with a second anti-DNA monoclonal antibody coupled to peroxidase. Nucleosome levels were quantified by determining the amount of peroxidase retained in the immunocomplex. Peroxidase activity was determined photometrically at $405 \mathrm{~nm}$ using ABTS [2,2'-azino-di(3-ethylbenzthiazoline-6-sulfonate)] as the substrate.

Measurement of ROS. The fluorescent probe, chloromethyl2,7-dichlorofluorescein diacetate (DCFDA; Molecular Probes, Eugene, OR, USA), was used to measure intracellular ROS levels (27). Osteoblastic MC3T3-E1 cells were cultured for $24 \mathrm{~h}$ in $\alpha$-MEM containing $0.5 \% \mathrm{FBS}$, rinsed twice with DPBS, and then treated with $10 \mu \mathrm{M}$ of DCFDA for $1 \mathrm{~h}$. Cells were then rinsed, scraped, and their fluorescence was measured (excitation $485 \mathrm{~nm}$, emission $515 \mathrm{~nm}$ ) using a Zenyth 3100 multimode detector.

Determination of mitochondrial membrane potential $(\Delta \Psi m)$. The $\Delta \Psi_{\mathrm{m}}$ of cells was measured using a JC-1 (5,5',6,6'-tetrachloro-1,1',3,3'-tetraethylbenzimidazolylcarbocyanine iodide) $\Delta \Psi_{\mathrm{m}}$ detection kit (Cayman Chemical Co., Ann Arbor, MI, USA). Cells were incubated with the $\Delta \Psi_{\mathrm{m}}$-sensitive fluorescent dye, JC-1 for $20 \mathrm{~min}$ at $37^{\circ} \mathrm{C}$, washed twice in DPBS, and then red fluorescence (excitation $550 \mathrm{~nm}$, emission $600 \mathrm{~nm}$ ) and green fluorescence (excitation $485 \mathrm{~nm}$, emission $535 \mathrm{~nm}$ ) were measured using a Zenyth 3100 multimode detector. 
Table I. Primer sequences used in this study.

\begin{tabular}{llll}
\hline Gene & Accession no. & \multicolumn{1}{c}{ Forward primer } & \multicolumn{1}{c}{ Reverse primer } \\
\hline AKT1 & NM_009652.3 & 5'-TCG TGT GGC AGG ATG TGT AT-3' & 5'-ACC TGG TGT CAG TCT CAG AGG-3' \\
AKT2 & NM_001110208.1 & 5'-CGA CCC AAC ACC TTT GTC A-3' & 5'-GAT AGC CCG CAT CCA CTC T-3' \\
AKT3 & NM_011785.3 & 5'-TGG ACC ACT GTT ATA GAG AGA ACA TTT-3' & 5'-TGG ATA GCT TCC GTC CAC TC-3' \\
ALP & NM_007431.2 & 5'-GGC CAG CTA CAC CAC AAC A-3' & 5'-CTG AGC GTT GGT GTT ATA TGT CTT-3' \\
BMP2 & NM_007553.2 & 5'-GGT CAC AGA TAA GGC CAT TGC-3' & 5'-GCT TCC GCT GTT TGT GTT TG-3' \\
BMP4 & NM_007554.2 & 5'-GAG GAG TTT CCA TCA CGA AGA-3' & 5'-GCT CTG CCG AGG AGA TCA-3' \\
BMP7 & NM_007557.2 & 5'-CGA TAC CAC CAT CGG GAG TTC-3' & 5'-AAG GTC TCG TTG TCA AAT CGC-3' \\
Collagen & NM_007742.3 & 5'-AGA CAT GTT CAG CTT TGT GGA C-3' & 5'-GCA GCT GAC TTC AGG GAT G-3' \\
GPX1 & NM_008160.6 & 5'-GGT TTC CCG TGC AAT CAG T-3' & 5'-TCG GAC GTA CTT GAG GGA AT-3' \\
GPX4 & NM_001037741.2 & 5'-TAA GAA CGG CTG CGT GGT-3' & 5'-GTA GGG GCA CAC ACT TGT AGG-3' \\
OPG & NM_008764.3 & 5'-ATG AAC AAG TGG CTG TGC TG-3' & 5'-CAG TTT CTG GGT CAT AAT GCA A-3' \\
OPN & NM_001204201.1 & 5'-TGA GAT TGG CAG TGA TTT GC-3' & 5'-ATC TGG GTG CAG GCT GTA AA-3' \\
Osteocalcin & NM_031368.4 & 5'-CAC CAT GAG GAC CCT CTC TC-3' & 5'-TGG ACA TGA AGG CTT TGT CA-3' \\
PI3K & NM_020272.2 & 5'-TTT GGG AGA CTG AAT CTC TGG-3' & 5'-GTG GCA TCC TTT ACA ATC TCG-3' \\
SOD1 & NM_011434.1 & 5'-CCA TCA GTA TGG GGA CAA TAC A-3' & 5'-GGT CTC CAA CAT GCC TCT CT-3' \\
SOD2 & NM_013671.3 & 5'-GAC CCA TTG CAA GGA ACA A-3' & 5'-GTA GTA AGC GTG CTC CCA CAC-3' \\
SOD3 & NM_011435.3 & 5'-GGG GAG GCA ACT CAG AGG-3' & 5'-TGG CTG AGG TTC TCT GCA C-3' \\
\hline
\end{tabular}

Mitochondrial depolarization (i.e., loss of $\Delta \Psi_{\mathrm{m}}$ ) was indicated by a decrease in the red/green fluorescence ratio.

Alkaline phosphatase activity. At the time of cell harvesting, the medium was removed and the cell monolayer was gently washed twice with PBS. The cells were then lysed with $0.2 \%$ Triton X-100 and the lysate was centrifuged at $14,000 \mathrm{x} g$ for $5 \mathrm{~min}$. The cleared supernatant was used for the measurement of ALP activity and protein concentration. The ALP activity and protein concentration were determined using an ALP activity assay kit (Somang Co., Korea) and a Bradford assay kit (Bio-Rad, Hercules, CA, USA), respectively.

Collagen contents. Cellular collagen content was measured using a Sircol Collagen Assay kit (Biocolor Ltd., Carrickfergus, Northern Ireland, UK). This assay is a quantitative dye-binding method designed for the analysis of collagens extracted from mammalian tissues and cells during in vitro culture. The dye reagent binds specifically to the $[\mathrm{Gly}-\mathrm{X}-\mathrm{Y}]_{\mathrm{n}}$ helical structure found in mammalian collagens (types I-V).

RNA extraction. Total RNA was isolated from cells using the TRIzol reagent (Invitrogen). After isolation, RNA integrity was assessed using an Agilent 2100 Bioanalyzer (Agilent Technologies, Palo Alto, CA, USA). The cDNAs were synthesized with the Transcriptor first strand cDNA synthesis kit (Roche Diagnostics GmbH, Mannheim, Germany) and stored at $-70^{\circ} \mathrm{C}$ until further processing. All procedures were performed according to the manufacturer's instructions.

Real-time RT-PCR. Real-time PCR was performed to verify the differential expression of selected genes using a Roche LightCycler 480 System (Roche Diagnostics $\mathrm{GmbH}$ ) and the TaqMan method using the Roche Universal Probe Library (UPL) kit. Relative gene expression was determined by employing the comparative $\mathrm{CT}$ method. All reactions were carried out in a total volume of $20 \mu \mathrm{l}$ of reaction mixture containing $10.0 \mu \mathrm{l}$ of $2 \mathrm{X}$ UPL master mix, $1.0 \mu \mathrm{l}$ of $5^{\prime}$ primer (10 pmol $/ \mu \mathrm{l}), 1.0 \mu \mathrm{l}$ of $3^{\prime}$ primer $(10 \mathrm{pmol} / \mathrm{ml}), 0.2 \mu \mathrm{l}$ of UPL probe, $1.0 \mu \mathrm{l}$ of cDNA and $6.8 \mu \mathrm{l}$ of sterile water. The thermal cycling conditions for PCR were an initial denaturation for $10 \mathrm{~min}$ at $95^{\circ} \mathrm{C}$, followed by 40 cycles of $94^{\circ} \mathrm{C}$ for $10 \mathrm{sec}$ and $60^{\circ} \mathrm{C}$ for $30 \mathrm{sec}$. The primers summarized in Table I were designed by the Roche ProbeFinder assay tool. For the RT-PCR analysis, duplicate PCRs were carried out for each cDNA. Negative controls (except templates) were included in the PCR reaction to ensure specific amplification. LightCycler 480 software version 1.2 (Roche Diagnostics $\mathrm{GmbH}$ ) was used for the analysis of the quantitative PCR. The values obtained from each sample were normalized to HPRT (hypoxanthine guanine phosphoribosyl transferase) expression. Levels of each gene expression in all experimental groups were compared to the expression levels of the control group.

Statistical analysis. The results are expressed as the means \pm SD. Statistical analysis was performed using one-way ANOVA with a subsequent Tukey's multiple comparison test. A P-value $<0.05$ was considered to indicate statistically significant differences. Statistical analysis was performed using SAS software (SAS Institute Inc., Cary, NC, USA).

\section{Results}

Glabridin inhibits dRib-induced decrease in MC3T3-E1 osteoblastic cell survival. To evaluate the effect of glabridin itself on MC3T3-E1 osteoblastic cell survival, cells were incubated in $\alpha$-MEM containing $0.5 \%$ FBS with increasing concentrations of glabridin $(0.01-50 \mu \mathrm{M})$ for $24 \mathrm{~h}$ and then cell viability was determined using the CCK- 8 assay. Glabridin at concentrations of $0.01-5 \mu \mathrm{M}$ had no effect on cell viability, 
A

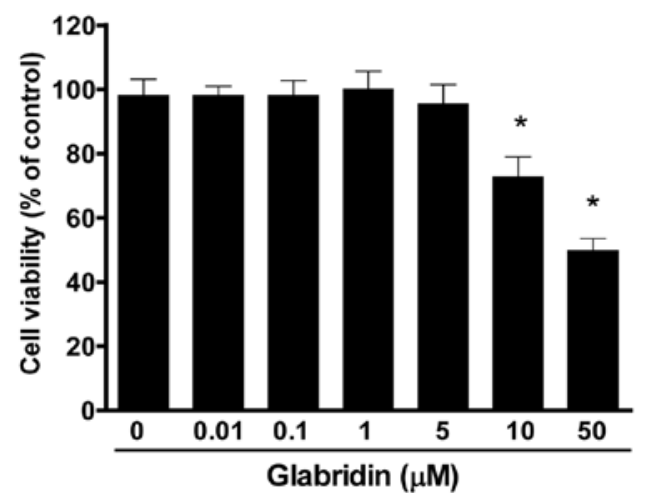

B

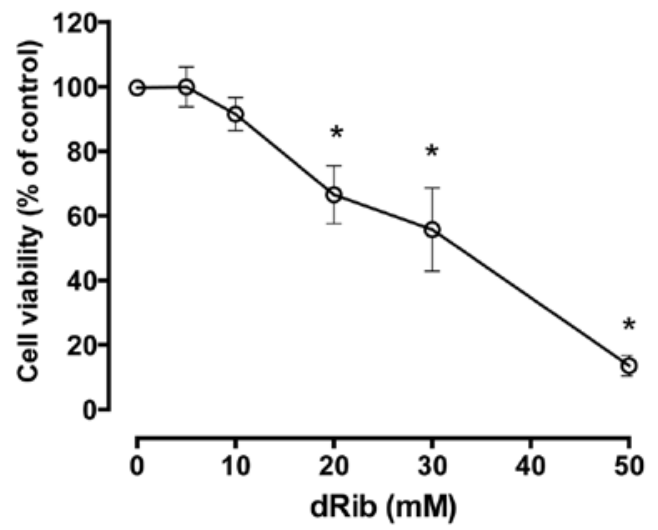

C

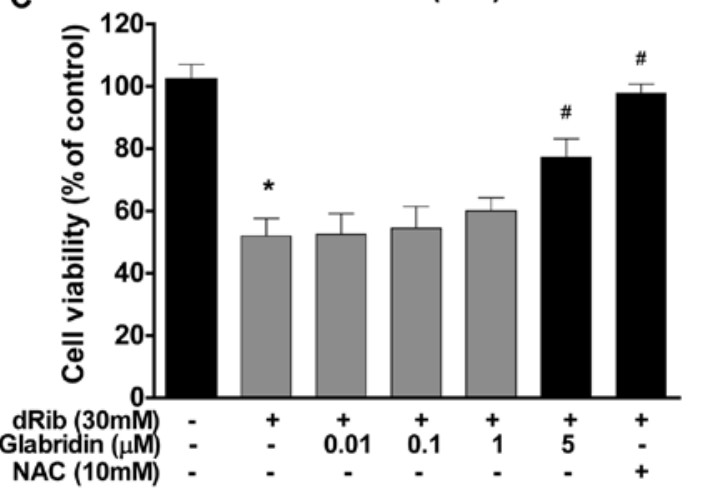

Figure 1. Protective effects of glabridin on dRib-induced cell viability. (A) Effect of glabridin on cell viability in osteoblastic cells. Cells were cultured with an increasing concentration of glabridin. (B) Cell viability treated with different concentrations of dRib. Cells were cultured in RPMI1640 medium containing $0.5 \%$ FBS for $24 \mathrm{~h}$ in the presence of an increasing concentration of dRib. (C) Cells were preincubated with glabridin or NAC for $30 \mathrm{~min}$ at the indicated concentrations and then cultured with $30 \mathrm{mM}$ $\mathrm{dRib}$ for $24 \mathrm{~h}$. Cell viability was determined using the CCK- 8 assay. The data are expressed as the means \pm SD of four independent determinations, each performed in quadruplicate. ${ }^{*} \mathrm{P}<0.05$ vs. untreated control; ${ }^{*} \mathrm{P}<0.05$ vs. dRib-treated cells.

while higher doses $(>10 \mu \mathrm{M})$ were found to be cytotoxic in a dose-dependent manner (Fig. 1A). Therefore, we chose the highest non-toxic concentration of glabridin $(5 \mu \mathrm{M})$ for all subsequent cell culture experiments. To evaluate the effect of dRib on MC3T3-E1 osteoblastic cell survival, cells were incubated for $24 \mathrm{~h}$ in $\alpha$-MEM containing $0.5 \%$ FBS with increasing concentrations of dRib $(10-50 \mathrm{mM})$. We observed a dose-dependent decrease in cell viability in cells exposed to various concentrations of $\mathrm{dRib}$ for $24 \mathrm{~h}$ (Fig. 1B). Based on the results of these cytotoxicity studies, we used $30 \mathrm{mM}$ of $\mathrm{dRib}$ in subsequent biochemical assays. At this concentration, $~ 50 \%$
A

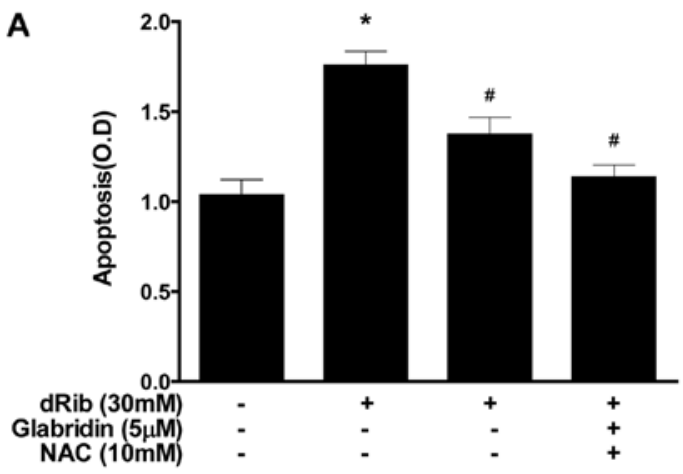

B

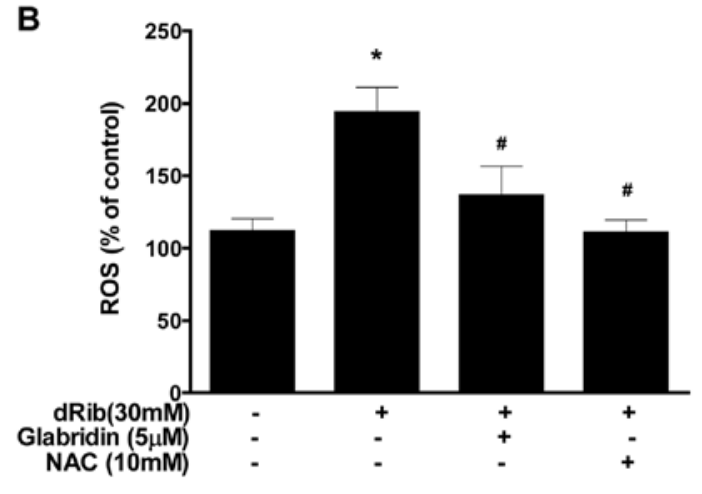

C

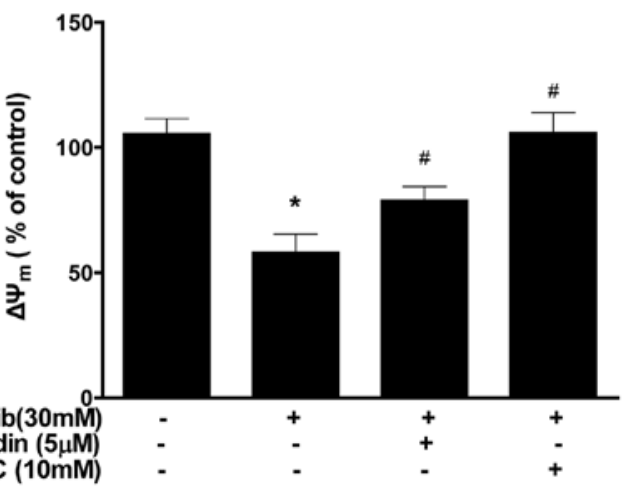

Figure 2. Effect of glabridin on (A) dRib-induced apoptosis, (B) ROS production, and (C) $\Delta \Psi_{\mathrm{m}}$ in osteoblastic cells. Cells were preincubated with $5 \mu \mathrm{M}$ glabridin or $10 \mathrm{mM} \mathrm{NAC}$ for $30 \mathrm{~min}$ and then cultured with $30 \mathrm{mM}$ dRib for $24 \mathrm{~h}$. The data are expressed as the means \pm SD of three independent determinations, each performed in quadruplicate. ${ }^{*} \mathrm{P}<0.05$ vs. untreated control; ${ }^{\#} \mathrm{P}<0.05$ vs. dRib-treated cells.

inhibition of cell viability occurred in $24 \mathrm{~h}$ under our experimental culture conditions. To determine whether glabridin had an effect on the dRib-induced decrease in cell survival, cells were preincubated with glabridin for $30 \mathrm{~min}$ and then cultured in the presence of $30 \mathrm{mM}$ of $\mathrm{dRib}$ for $24 \mathrm{~h}$. The CCK- 8 assays revealed that glabridin $(5 \mu \mathrm{M})$ partially reversed the dRibmediated reduction in cell viability (Fig. 1C). The antioxidant $\mathrm{N}$-acetyl-L-cysteine (NAC) was used to investigate the mechanism of dRib-induced cell damage. Pretreatment of cells with $10 \mathrm{mM}$ of NAC almost completely reversed the dRib-induced cytotoxicity.

Effect of glabridin on the dRib-induced ROS generation, apoptosis and intrinsic $\Delta \Psi_{m}$. The oxidative stress caused by dRib in MC3T3-E1 osteoblastic cells was evaluated by measuring ROS generation, apoptosis and $\Delta \Psi_{\mathrm{m}}$. Oxidative 

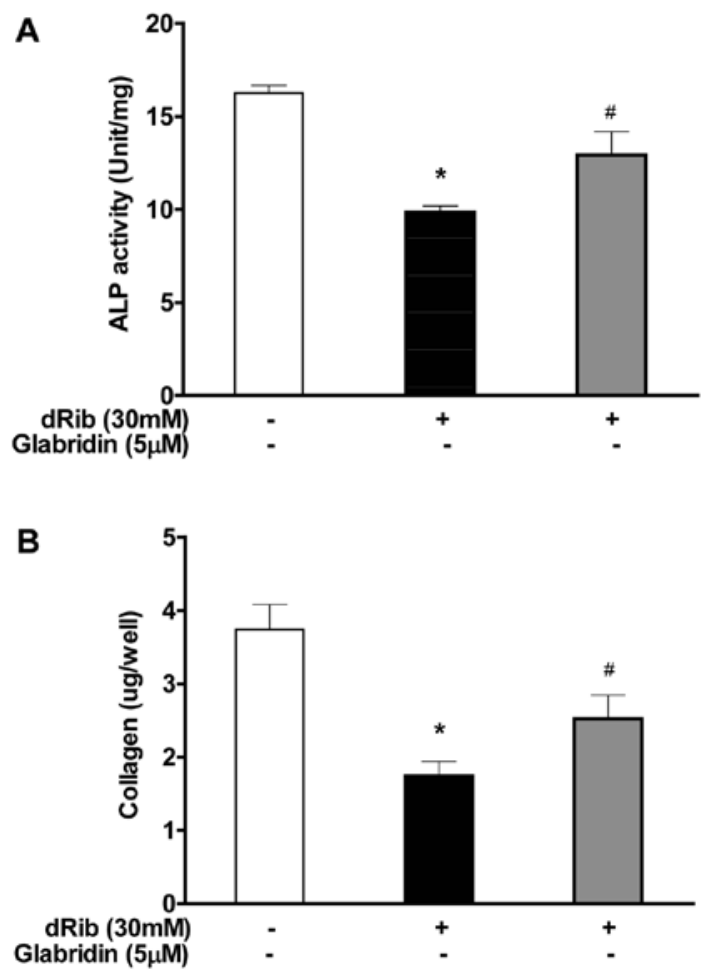

Figure 3. Effects of glabridin on (A) dRib-induced ALP activity and (B) collagen contents. Cells were preincubated with $5 \mu \mathrm{M}$ glabridin and then cultured with $30 \mathrm{mM}$ dRib. ALP activity and collagen contents were assessed as described in Materials and methods. The data are expressed as the means \pm SD of three independent determinations, each performed in quadruplicate. ${ }^{*} \mathrm{P}<0.05$ vs. untreated control; ${ }^{*} \mathrm{P}<0.05$ vs. dRib-treated cells.

stress may initiate a mitochondrial permeability transition event, which is an early mediator of cellular apoptosis. When cells were treated with $30 \mathrm{mM}$ of $\mathrm{dRib}$, an increase in ROS generation and apoptosis was observed, while a decrease in $\Delta \Psi_{\mathrm{m}}$ was noted. Treatment with glabridin $(5 \mu \mathrm{M})$ in the presence of $30 \mathrm{mM}$ of $\mathrm{dRib}$ attenuated all the $\mathrm{dRib}$-induced effects (Fig. 2). We used the antioxidant NAC to investigate the effect of oxidative stress in the cells. It was observed that NAC prevented the dRib-induced cellular effects.

Glabridin inhibits dRib-induced decrease in ALP activity and collagen contents in MC3T3-E1 osteoblastic cells. ALP activity and collagen content were measured to study the effect of glabridin on the osteoblastic cell differentiation. The reducing sugar $\mathrm{dRib}$ was found to have an inhibitory effect on osteoblastic differentiation markers, however, when osteoblasts were treated with $5 \mu \mathrm{M}$ of glabridin in the presence of $30 \mathrm{mM}$ of $\mathrm{dRib}$, significant increase in the major osteoblast-specific ALP activity and collagen contents was observed (Fig. 3).

Glabridin inhibits dRib-induced decrease in differentiation genes in MC3T3-E1 osteoblastic cells. To study the differentiated function of the osteoblastic cells at the transcriptional level, we analyzed the gene expression of a number of molecular markers of osteoblast differentiation. Five differentiation markers [ALP, collagen, osteopontin (OPN), osteoprotegerin $(\mathrm{OPG})$ and osteocalcin $(\mathrm{OC})]$ were observed to be downregulated in response to the dRib induction, however, treatment with

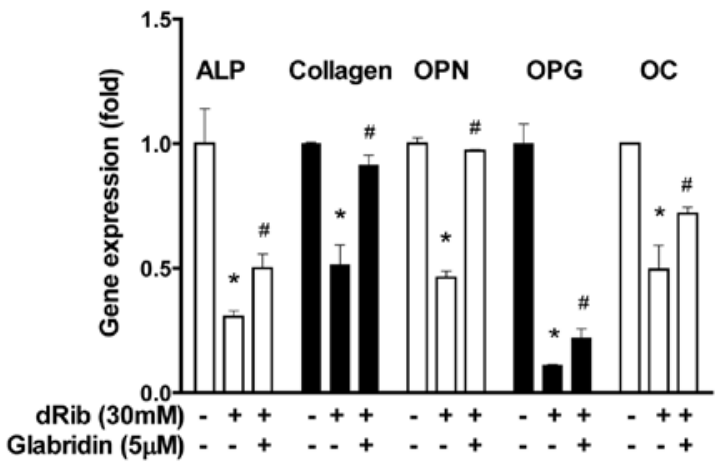

Figure 4. Effect of glabridin on dRib-induced gene expression involved in osteoblastic differentiation. Total RNA was extracted from osteoblastic cells and the mRNA levels for ALP, collagen, OPN, OPG and OC were assessed by real-time PCR as described in Materials and methods. The data are expressed as the means $\pm \mathrm{SD}$ of three independent determinations. ${ }^{*} \mathrm{P}<0.05$ vs. untreated control; ${ }^{\#} \mathrm{P}<0.05$ vs. dRib-treated cells.

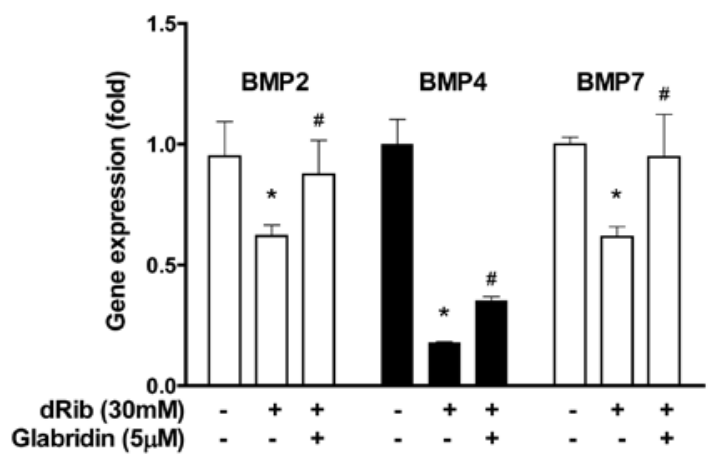

Figure 5. Effect of glabridin on dRib-induced BMP gene expression in osteoblastic cells. Total RNA was extracted from osteoblastic cells and the mRNA levels for BMP2, BMP4 and BMP7 were assessed by real-time PCR as described in Materials and methods. The data are expressed as the means $\pm \mathrm{SD}$ of three independent determinations. ${ }^{*} \mathrm{P}<0.05$ vs. untreated control; ${ }^{\#} \mathrm{P}<0.05$ vs. dRib-treated cells.

$5 \mu \mathrm{M}$ of glabridin partially inhibited dRib-induced downregulation of gene expression of differentiation markers (Fig. 4).

Glabridin inhibits dRib-induced decrease in bone morphogenetic protein (BMP) genes in MC3T3-E1 osteoblastic cells. BMPs are known to be the most potent regulators of osteoblastic differentiation among numerous local factors. The reducing sugar $\mathrm{dRib}$ was found to have an inhibitory effect on the gene expression of BMPs. However, when osteoblasts were treated with $5 \mu \mathrm{M}$ of glabridin in the presence of $30 \mathrm{mM}$ of dRib, BMPs including BMP2, BMP4 and BMP7 were significantly increased (Fig. 5).

Glabridin inhibits dRib-induced decrease in phosphatidylinositol 3'-kinase (PI3K) and AKT2 genes in MC3T3-E1 osteoblastic cells. We examined the effect of glabridin on the PI3K/AKT genes, which were involved in cellular survival pathways. When osteoblasts were treated with $30 \mathrm{mM}$ of dRib, the gene expression of PI3K, AKT1, AKT2 and AKT3 was downregulated. However, treatment with $5 \mu \mathrm{M}$ of glabridin in the presence of $30 \mathrm{mM}$ dRib increased the gene expression of PI3K and AKT2 but not AKT1, AKT3 (Fig. 6). 


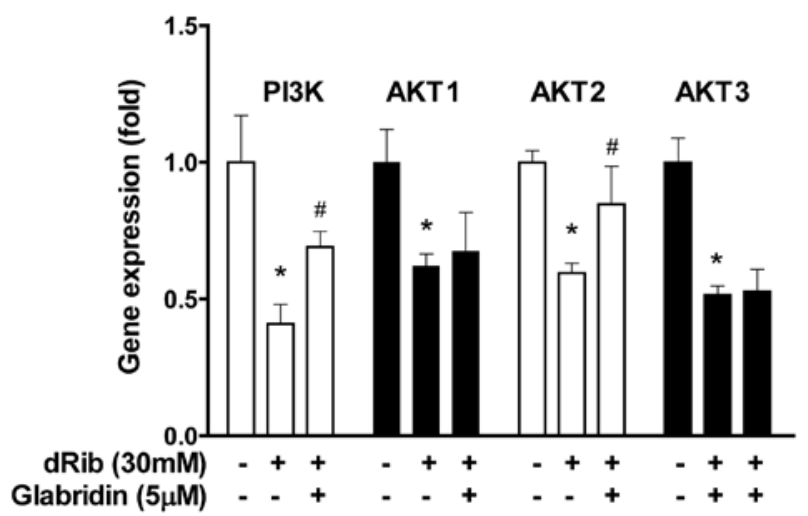

Figure 6. Effect of glabridin on dRib-induced gene expression involved in osteoblastic survival signaling pathway. Total RNA was extracted from osteoblastic cells and the mRNA levels for PI3K, AKT1, AKT2 and AKT3 were assessed by real-time PCR as described in Materials and methods. The data are expressed as the means \pm SD of three independent determinations. ${ }^{*} \mathrm{P}<0.05$ vs. untreated control; ${ }^{*} \mathrm{P}<0.05$ vs. dRib-treated cells.

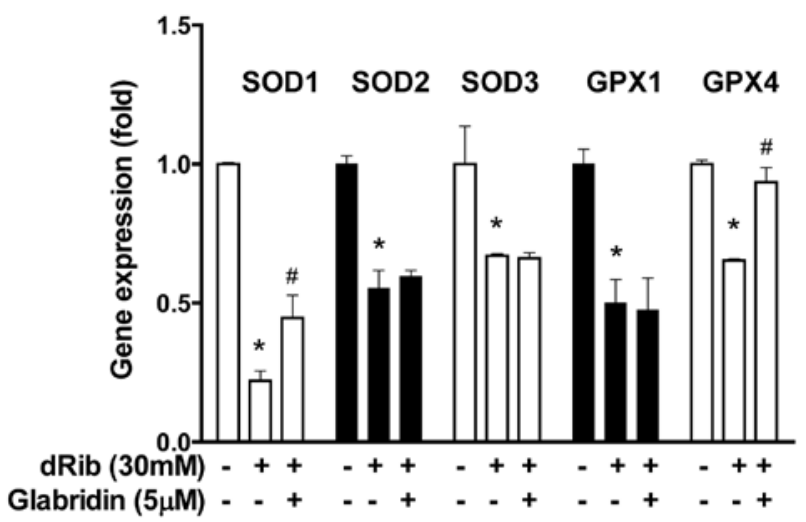

Figure 7. Effect of glabridin on dRib-induced gene expression involved in osteoblastic antioxidant enzymes. Total RNA was extracted from osteoblastic cells and the mRNA levels for SOD, SOD2, SOD3, GPX1 and GPX4 were assessed by real-time PCR as described in Materials and methods. The data are expressed as the means \pm SD of three independent determinations. ${ }^{*} \mathrm{P}<0.05$ vs. untreated control; ${ }^{\#} \mathrm{P}<0.05$ vs. dRib-treated cells.

Glabridin inhibits dRib-induced decrease in antioxidant enzyme gene in MC3T3-E1 osteoblastic cells. In addition to the biochemical aspects of oxidative stress, the gene expression of antioxidative enzymes was investigated. When osteoblasts were treated with $30 \mathrm{mM}$ of $\mathrm{dRib}$, gene expression of SOD1, SOD2, SOD3, GPX1 and GPX4 was downregulated. However, treatment with $5 \mu \mathrm{M}$ of glabridin in the presence of $30 \mathrm{mM}$ of $\mathrm{dRib}$ led to an increase in the gene expression of SOD1 and GPX4, but not SOD2, SOD3, or GPX1 (Fig. 7).

\section{Discussion}

In the present study, we investigated the effects of glabridin on dRib-induced oxidative damage and cellular dysfunction using an MC3T3-E1 osteoblastic cell culture model. The $\mathrm{dRib}$ is a strong reducing sugar that is highly reactive with proteins, in contrast to glucose which has the lowest reducing capacity of all the monosaccharides (9). Therefore, we chose $\mathrm{dRib}$ as a surrogate for glucose to study the effects of oxida- tive stress in osteoblastic cells. One of the mechanisms of diabetes-related bone disease may be the direct effects of high glucose on osteoblastic cells. This hypothesis is explained by in vitro studies, which state that hyperglycemia has been shown to inhibit osteoblastic cell proliferation and differentiation $(28,29)$, indicating that extracellular high glucose would directly impair osteoblastic functions resulting in bone disease. In our previous studies, we found that $\mathrm{dRib}$ induces cellular damage in pancreatic $\beta$-cells by increasing oxidative stress and protein glycation (12-15). Recently, we also reported that dRib induces cellular dysfunction and apoptosis in the MC3T3-E1 mouse osteoblastic cell line by increasing oxidative stress (18). In this study, glabridin partially reversed the dRib-mediated reduction in cell viability (Fig. 1). The antioxidant NAC was used to investigate the mechanism of dRib-induced cell damage. Pretreatment of cells with NAC almost completely reversed the dRib-induced cytotoxicity. These findings suggest that the dRib-induced cytotoxicity was most likely due to oxidative stress-induced effects. Our recent finding showed that the antioxidants NAC and $\alpha$-lipoic acid (ALA) almost reversed the dRib-mediated reduction in viability of MC3T3-E1 osteoblastic cells (18). When cells were treated with dRib, ROS generation and apoptosis increased, while $\Delta \Psi_{\mathrm{m}}$ decreased. Treatment with glabridin in the presence of $\mathrm{dRib}$ attenuated all the dRib-induced effects (Fig. 2). The antioxidant NAC prevented the dRib-induced cellular effects. These data are consistent with our previous studies, which stated that the antioxidants NAC and ALA protected pancreatic $\beta$-cells and MC3T3-E1 osteoblastic cells against oxidative stress as shown in terms of reduction in ROS generation and apoptosis $(12-14,18,27)$. These findings indicate that glabridin can function as an antioxidant and thereby protect MC3T3-E1 osteoblastic cells from dRibinduced oxidative cell damage. We used JC-1 staining to assess $\Delta \Psi_{\mathrm{m}}$, which is a marker of mitochondrial oxidative phosphorylation activity. The JC-1 is a lipophilic and cationic dye that permeates plasma and mitochondrial membranes of cells. A low JC-1 ratio indicates a low amount of the aggregated form of JC-1 in the mitochondria, which correlates with a high amount of ROS (30). Oxidative stress may initiate a mitochondrial permeability transition event, which is an early indication of the initiation of cellular apoptosis. This process is typically defined as a collapse in the electrochemical gradient across the mitochondrial membrane, as measured by the change in the $\Delta \Psi_{\mathrm{m}}(31)$. Mitochondrial dysfunction is a consequence of oxidative damage caused by increased oxidant levels. In our study, pretreatment of cells with glabridin attenuated marked decrease in dRib-induced $\Delta \Psi_{\mathrm{m}}$ (Fig. 2C). These results indicate that glabridin protects mitochondrial functions by functioning as an antioxidant in osteoblastic cells.

The present study demonstrated attenuation of dRibinduced decrease in major osteoblast differentiation markers, such as ALP activity and collagen contents, in the presence of glabridin (Fig. 3). The MC3T3-E1 cells are the osteoblastic precursor cell lines, which are cloned from calvariae of newborn mice (32); they are the most frequently used cell lines to study osteoblast differentiation, as well as the function of mature osteoblastic cells. Osteoblast differentiation is the primary event of bone formation. Bone ALP is a glycopro- 
tein localized in the plasma membrane of osteoblastic cells, which is also one of the osteoblastic phenotype markers (33). Alterations in this activity have been observed in osteoporosis and other metabolic bone diseases. High levels of ALP activity are shown in both preosteoblasts and osteoblasts in vivo and in differentiating osteoblasts in vitro. Osteoblast cells produce type I collagen, which is the most abundant protein in the bone matrix, serves an early marker of osteoblast differentiation, and is the major organic component of mineralized bone matrix (34).

In this study, five differentiation markers (ALP, collagen, OPN, OPG, OC) were found to be downregulated by dRib, however, glabridin partially inhibited dRib-induced downregulation of differentiation genes (Fig. 4). OPN is a major acidic phosphorylated glycoprotein secreted by osteoblasts and acts as a regulator of bone formation (35). OPG, produced by osteoblasts, is one of the regulators of bone metabolism and inhibits bone resorption by regulating function of osteoclast (36). OC is also known as a marker of bone formation and is the most abundant non-collagenous protein in bone (37). Thus, these molecular markers are important and are the best-known regulators of osteoblast function.

When osteoblasts were treated with glabridin in the presence of dRib, a significant increase in the bone morphogenetic proteins BMP2, BMP4 and BMP7 was observed (Fig. 5). The MC3T3-E1 cells are highly BMP-responsive and can complete differentiation processes in the long-term cultures. Previous studies have demonstrated that BMPs stimulate ALP activity, collagen synthesis, parathyroid hormone $(\mathrm{PTH})$ responsiveness, and osteocalcin production in osteoblastic cells (38-40), suggesting that BMPs stimulate differentiation function of osteoblastic cells. It has been suggested that glabridin exerts differentiation function of osteoblastic cells by stimulation of BMP production.

Glabridin was also found to induce the activation of PI3K and AKT2, which were inhibited by dRib (Fig. 6). Since these signalings are involved in cellular survival pathways, glabridin might be cytoprotective for osteoblastic cells during oxidative stress responses. The phosphatidylinositol 3'-kinase (PI3K)-AKT (protein kinase B) signaling pathway is known to be activated by many types of cellular stimuli or toxic insults and regulates fundamental cellular functions such as transcription, translation, proliferation, growth, and survival (41). One of the important functions of activated $\mathrm{PI} 3 \mathrm{~K}$ in cells is the inhibition of apoptosis (42). The AKT is a good candidate for mediating these PI3K-dependent cell survival responses. AKT has been implicated as an anti-apoptotic factor in many different cell death paradigms, including the withdrawal of extracellular signaling factors, oxidative and osmotic stress, irradiation, and the treatment of cells with chemotherapeutic drugs and ischemic shock (43). Zinc has been reported to inhibit hydrogen peroxide-induced MC3T3-E1 osteoblastic cells via the mitogen activated protein kinase (MAPK) and PI3K/AKT pathways. The various flavonoids including deoxyactein (44), honokiol (45), apocynin (46) have been demonstrated to impart protective effects against antimycin A (an inhibitor of mitochondrial electron transport)-induced oxidative cell damage via the activation of PI3K and/or AKT in MC3T3-E1 osteoblastic cells.
When osteoblasts were treated with glabridin in the presence of dRib, significant increases in the gene expression of SOD1 and GPX4 were observed (Fig. 7). In general, excess ROS must be promptly eliminated from the cell by a variety of antioxidant defense mechanisms. Cellular antioxidant enzymes and other redox molecules serve to counterbalance ROS generated in the cell. Superoxide dismutase (SOD), which catalyzes the dismutation of the superoxide anion into hydrogen peroxide and molecular oxygen, is one of the most important antioxidant enzymes (47). SOD enzymes are classified into three groups: CuZn-SOD (SOD1), which is located in the cytoplasm, Mn-SOD (SOD2), which is located in the mitochondria, and EC-SOD (SOD3), which is located in extracellular location. Glutathione peroxidase (GPx) catalyzes the reduction of hydroperoxides, including hydrogen peroxides, by reduced glutathione and functions to protect the cell from oxidative damage. Glutathione peroxidase 1 (GPX1) is the most abundant version, found in the cytoplasm of nearly all mammalian tissues, whose preferred substrate is hydrogen peroxide. GPX4 has a high preference for lipid hydroperoxides. It has been reported that various flavonoids increased the activity of antioxidant enzymes in osteoblastic cells. Quercetin can diminish oxidative human osteoblastic cell damage by scavenging the radicals and by upregulating the expression of heme oxygenase-1 (HO-1) and SOD-1 exposed to cigarette smoke medium (48). An extract of total flavonoids from persimmon leaves significantly decreased the level of reactive oxygen species (ROS) and malondialdehyde (MDA), while increasing the activity of catalase (CAT), SOD and GPX in MC3T3-E1 cells. Simvastatin abated oxidative stress through enhancing catalase, HO-1, and SOD activity and suppressing NADPH oxidase activity in an aged and ovariectomized rat model (49). Another study demonstrated that intracellular redox imbalance caused by SOD1 deficiency played a pivotal role in the development and progression of bone fragility both in vivo and in vitro (50).

In contrast to the antioxidant effects of glabridin in osteoblastic cells as noted in this study, glabridin has also been shown to confer anticancer effects in other cell types. Previously, it has been demonstrated that glabridin exhibited effective inhibition of cell metastasis by decreasing cancer cell migration and invasion of human non-small cell lung cancer A549 cells (51). Glabridin has also been reported to exhibit effective inhibition of cell metastasis by decreasing cancer cell migration and invasion of MDA-MB-231 human breast adenocarcinoma cells (52). Thus, glabridin appears to have different biological effects on different cell types. The mechanism through which glabridin exerts these cell-specific effects remains to be elucidated.

In summary, we have shown that glabridin attenuates dRib-induced cell damage in MC3T3-E1 osteoblastic cells due to its antioxidant activity and improves differentiation function, which may promote bone recovery in diabetes-related bone diseases.

\section{Acknowledgements}

This study was supported by the ACE program through the National Research Foundation of Korea (NRF) grant funded by the Korean Ministry of Education, Science and Technology (MEST) (no. 20110028203). 


\section{References}

1. Robertson RP, Harmon J, Tran PO, Tanaka Y and Takahashi H: Glucose toxicity in beta-cells: type 2 diabetes, good radicals gone bad, and the glutathione connection. Diabetes 52: 581-587, 2003.

2. Robertson RP: Chronic oxidative stress as a central mechanism for glucose toxicity in pancreatic islet beta cells in diabetes. J Biol Chem 279: 42351-42354, 2004.

3. López-Ibarra PJ, Pastor MM, Escobar-Jiménez F, Pardo MD, González AG, Luna JD, Requena ME and Diosdado MA: Bone mineral density at time of clinical diagnosis of adult-onset type 1 diabetes mellitus. Endocr Pract 7: 346-351, 2001.

4. Tuominen JT, Impivaara O, Puukka P and Ronnemaa T: Bone mineral density in patients with type 1 and type 2 diabetes. Diabetes Care 22: 1196-1200, 1999.

5. Herskind AM, Christensen K, Nørgaard-Andersen K and Andersen JF: Diabetes mellitus and healing of closed fractures. Diabetes Metab 18: 63-64, 1992

6. Bai XC, Lu D, Bai J, Zheng H, Ke ZY, Li XM and Luo SQ: Oxidative stress inhibits osteoblastic differentiation of bone cells by ERK and NF-kappaB. Biochem Biophys Res Commun 314 197-207, 2004.

7. Fatokun AA, Stone TW and Smith RA: Hydrogen peroxideinduced oxidative stress in MC3T3-E1 cells: the effects of glutamate and protection by purines. Bone 39: 542-551, 2006.

8. Seeman E: Reduced bone formation and increased bone resorption: rational targets for the treatment of osteoporosis. Osteoporos Int 14 (Suppl 3): S2-S8, 2003

9. Thornalley P, Wolff S, Crabbe J and Stern A: The autoxidation of glyceraldehyde and other simple monosaccharides under physiological conditions catalysed by buffer ions. Biochim Biophys Acta 797: 276-287, 1984.

10. Kaneto H, Fujii J, Myint T, Miyazawa N, Islam KN, Kawasaki Y, Suzuki K, Nakamura M, Tatsumi H, Yamasaki Y and Taniguchi N: Reducing sugars trigger oxidative modification and apoptosis in pancreatic beta-cells by provoking oxidative stress through the glycation reaction. Biochem J 320: 855-863, 1996.

11. Bunn HF and Higgins PJ: Reaction of monosaccharides with proteins: possible evolutionary significance. Science 213 : 222-224, 1981.

12. Koh G, Suh KS, Chon S, Oh S, Woo JT, Kim SW, Kim JW and Kim YS: Elevated cAMP level attenuates 2-deoxy-d-riboseinduced oxidative damage in pancreatic beta-cells. Arch Biochem Biophys 438: 70-79, 2005.

13. Koh G, Lee DH and Woo JT: 2-Deoxy-D-ribose induces cellular damage by increasing oxidative stress and protein glycation in a pancreatic beta-cell line. Metabolism 59: 325-332, 2010.

14. Lee YJ, Suh KS, Choi MC, Chon S, Oh S, Woo JT, Kim SW, Kim JW and Kim YS: Kaempferol protects HIT-T15 pancreatic beta cells from 2-deoxy-D-ribose-induced oxidative damage. Phytother Res 24: 419-423, 2010.

15. Suh KS, Oh S, Woo JT, Kim SW, Kim JW, Kim YS and Chon S: Apigenin attenuates 2-deoxy-D-ribose-induced oxidative cell damage in HIT-T15 pancreatic $\beta$-cells. Biol Pharm Bull 35: 121-126, 2012

16. Choi EM and Kim YH: Hesperetin attenuates the highly reducing sugar-triggered inhibition of osteoblast differentiation. Cell Biol Toxicol 24: 225-231, 2008.

17. Lee $\mathrm{KH}$ and Choi EM: Myricetin, a naturally occurring flavonoid, prevents 2-deoxy-D-ribose induced dysfunction and oxidative damage in osteoblastic MC3T3-E1 cells. Eur J Pharmacol 591: $1-6,2008$.

18. Suh KS, Choi EM, Kwon M, Chon S, Oh S, Woo JT, Kim SW, Kim JW and Kim YS: Kaempferol attenuates 2-deoxy-d-riboseinduced oxidative cell damage in MC3T3-E1 osteoblastic cells. Biol Pharm Bull 32: 746-749, 2009.

19. Chin YW, Jung HA, Liu Y, Su BN, Castoro JA, Keller WJ, Pereira MA and Kinghorn AD: Anti-oxidant constituents of the roots and stolons of licorice (Glycyrrhiza glabra). J Agric Food Chem 55: 4691-4697, 2007.

20. Fukai T, Sakagami H, Toguchi M, Takayama F, Iwakura I Atsumi T, Ueha T, Nakashima $H$ and Nomura T: Cytotoxic activity of low molecular weight polyphenols against human oral tumor cell lines. Anticancer Res 20: 2525-2536, 2000

21. Fukai T, Marumo A, Kaitou K, Kanda T, Terada S and Nomura T: Anti-Helicobacter pylori flavonoids from licorice extract. Life Sci 71: 1449-1463, 2002.
22. Haraguchi H, Yoshida N, Ishikawa H, Tamura Y, Mizutani K and Kinoshita T: Protection of mitochondrial functions against oxidative stresses by isoflavans from Glycyrrhiza glabra. J Pharm Pharmacol 52: 219-223, 2000.

23. Choi EM: The licorice root derived isoflavan glabridin increases the function of osteoblastic MC3T3-E1 cells. Biochem Pharmacol 70: 363-368, 2005.

24. Choi EM: Glabridin protects osteoblastic MC3T3-E1 cells against antimycin $\mathrm{A}$ induced cytotoxicity. Chem Biol Interact 193: 71-78, 2011

25. Kim HS, Suh KS, Sul D, Kim BJ, Lee SK and Jung WW: The inhibitory effect and the molecular mechanism of glabridin on RANKL-induced osteoclastogenesis in RAW264.7 cells. Int J Mol Med 29: 169-177, 2012

26. Kanno S, Anuradha CD and Hirano S: Localization of zinc after in vitro mineralization in osteoblastic cells. Biol Trace Elem Res 83: 39-47, 2001.

27. Suh KS, Chon S, Oh S, Kim SW, Kim JW, Kim YS and Woo JT: Prooxidative effects of green tea polyphenol (-)-epigallocatechin3-gallate on the HIT-T15 pancreatic beta cell line. Cell Biol Toxicol 26: 189-199, 2010

28. Balint E, Szabo P, Marshall CF and Sprague SM: Glucose-induced inhibition of in vitro bone mineralization. Bone 28: 21-28, 2001.

29. Terada M, Inaba M, Yano Y, Hasuma T, Nishizawa Y, Morii H and Otani S: Growth-inhibitory effect of a high glucose concentration on osteoblast-like cells. Bone 22: 17-23, 1998.

30. Szilágyi G, Simona L, Koska P, Telek G and Nagy Z: Visualization of mitochondrial membrane potential and reactive oxygen species via double staining. Neurosci Lett 399: 206-209, 2006.

31. Salido M, Gonzalez JL and Vilches J: Loss of mitochondrial membrane potential is inhibited by bombesin in etoposideinduced apoptosis in PC-3 prostate carcinoma cells. Mol Cancer Ther 6: 1292-1299, 2007.

32. Sudo H, Kodama HA, Amagai Y, Yamamoto S and Kasai S: In vitro differentiation and calcification in a new clonal osteogenic cell line derived from newborn mouse calvaria. J Cell Biol 96: 191-198, 1983.

33. Bellows CG, Aubin JE and Heersche JN: Initiation and progression of mineralization of bone nodules formed in vitro: the role of alkaline phosphatase and organic phosphate. Bone Miner 14: 27-40, 1991.

34. Domon S, Shimokawa H, Yamaguchi S and Soma K: Temporal and spatial mRNA expression of bone sialoprotein and type I collagen during rodent tooth movement. Eur J Orthod 23: 339-348, 2001

35. Chen Y, Bal BS and Gorski JP: Calcium and collagen binding properties of osteopontin, bone sialoprotein, and bone acidic glycoprotein-75 from bone. J Biol Chem 267: 24871-24878, 1992.

36. Khosla S: Minireview: the OPG/RANKL/RANK system. Endocrinology 142: 5050-5055, 2001.

37. Price PA and Nishimoto SK: Radioimmunoassay for the vitamin $\mathrm{K}$-dependent protein of bone and its discovery in plasma. Proc Natl Acad Sci USA 77: 2234-2238, 1980.

38. Takuwa Y, Ohse C, Wang EA, Wozney JM and Yamashita K: Bone morphogenetic protein-2 stimulates alkaline phosphatase activity and collagen synthesis in cultured osteoblastic cells, MC3T3-E1. Biochem Biophys Res Commun 174: 96-101, 1991.

39. Nakase T, Takaoka K, Masuhara K, Shimizu K, Yoshikawa H and Ochi T: Interleukin-1 $\beta$ enhances and tumor necrosis factor- $\alpha$ inhibits bone morphogenetic protein-2-induced alkaline phosphatase activity in MC3T3-E1 osteoblastic cells. Bone 21: 17-21, 1997.

40. Vivanco I and Sawyers CL: The phosphatidylinositol 3-Kinase AKT pathway in human cancer. Nat Rev Cancer 2: 489-501, 2002.

41. Yao R and Cooper GM: Requirement for phosphatidylinositol-3 kinase in the prevention of apoptosis by nerve growth factor. Science 267: 2003-2006, 1995.

42. Franke TF, Kaplan DR and Cantley LC: PI3K: downstream AKTion blocks apoptosis. Cell 88: 435-437, 1997.

43. Choi EM: Deoxyactein isolated from Cimicifuga racemosa protects osteoblastic MC3T3-E1 cells against antimycin A-induced cytotoxicity. J Appl Toxicol: Dec 19, 2011 (Epub ahead of print). doi: 10.1002/jat.1784,2011.

44. Choi EM: Honokiol protects osteoblastic MC3T3-E1 cells against antimycin A-induced cytotoxicity. Inflamm Res 60 1005-1012, 2011.

45. Choi EM and Lee YS: Protective effect of apocynin on antimycin A-induced cell damage in osteoblastic MC3T3-E1 cells. J Appl Toxicol: May 2, 2011 (Epub ahead of print). doi: 10.1002/jat.1689, 2011. 
46. Zelko IN, Mariani TJ and Folz RJ: Superoxide dismutase multigene family: a comparison of the CuZn-SOD (SOD1), Mn-SOD (SOD2), and EC-SOD (SOD3) gene structures, evolution, and expression. Free Radic Biol Med 33: 337-349, 2002.

47. Braun KF, Ehnert S, Freude T, Egaña JT, Schenck TL, Buchholz A, Schmitt A, Siebenlist S, Schyschka L, Neumaier M, Stöckle U and Nussler AK: Quercetin protects primary human osteoblasts exposed to cigarette smoke through activation of the antioxidative enzymes HO-1 and SOD-1. Sci World J 11: 23482357, 2011.

48. Sun L, Zhang J, Lu X, Zhang L and Zhang Y: Evaluation to the antioxidant activity of total flavonoids extract from persimmon (Diospyros kaki L.) leaves. Food Chem Toxicol 49: 2689-2696, 2011.

49. Yin H, Shi ZG, Yu YS, Hu J, Wang R, Luan ZP and Guo DH: Protection against osteoporosis by statins is linked to a reduction of oxidative stress and restoration of nitric oxide formation in aged and ovariectomized rats. Eur J Pharmacol 674: 200-206, 2012 .
50. Nojiri H, Saita Y,Morikawa D, Kobayashi K, Tsuda C, Miyazaki T, Saito M, Marumo K, Yonezawa I, Kaneko K, Shirasawa T and Shimizu T: Cytoplasmic superoxide causes bone fragility owing to low-turnover osteoporosis and impaired collagen cross-linking. J Bone Miner Res 26: 2682-2694, 2011.

51. Tsai YM, Yang CJ, Hsu YL, Wu LY, Tsai YC, Hung JY, Lien CT, Huang MS and Kuo PL: Glabridin inhibits migration, invasion, and angiogenesis of human non-small cell lung cancer A549 cells by inhibiting the FAK/rho signaling pathway. Integr Cancer Ther 10: 341-349, 2011.

52. Hsu YL, Wu LY, Hou MF, Tsai EM, Lee JN, Liang HL, Jong YJ, Hung CH and Kuo PL: Glabridin, an isoflavan from licorice root, inhibits migration, invasion and angiogenesis of MDA-MB-231 human breast adenocarcinoma cells by inhibiting focal adhesion kinase/Rho signaling pathway. Mol Nutr Food Res 55: 318-327, 2011. 